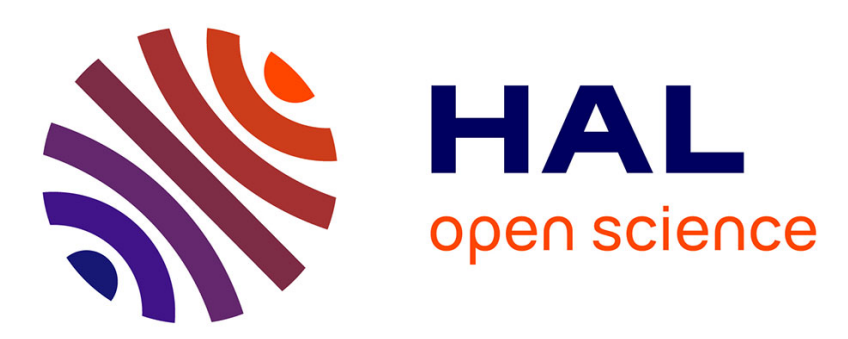

\title{
Lehmann rotation of cholesteric droplets subjected to a temperature gradient: role of the concentration of chiral molecules
}

Patrick Oswald

\section{- To cite this version: \\ Patrick Oswald. Lehmann rotation of cholesteric droplets subjected to a temperature gradient: role of the concentration of chiral molecules. European Physical Journal E: Soft matter and biological physics, 2009, 28 (4), pp.377-383. ensl-00507795}

\section{HAL Id: ensl-00507795}

\section{https://hal-ens-lyon.archives-ouvertes.fr/ensl-00507795}

Submitted on 3 Aug 2010

HAL is a multi-disciplinary open access archive for the deposit and dissemination of scientific research documents, whether they are published or not. The documents may come from teaching and research institutions in France or abroad, or from public or private research centers.
L'archive ouverte pluridisciplinaire HAL, est destinée au dépôt et à la diffusion de documents scientifiques de niveau recherche, publiés ou non, émanant des établissements d'enseignement et de recherche français ou étrangers, des laboratoires publics ou privés. 


\title{
Lehmann rotation of cholesteric droplets subjected to a
}

\section{temperature gradient: role of the concentration of chiral}

\section{molecules}

\section{P. Oswald ${ }^{a}$}

Laboratoire de Physique, École Normale Supérieure de Lyon, UMR 5672 of the CNRS, 46 Allée d'Italie, 69364 Lyon, France

Received:

\begin{abstract}
We present a systematic study of the Lehmann rotation of cholesteric droplets subjected to a temperature gradient when the concentration of chiral molecules is changed. The liquid crystal chosen is an eutectic mixture of $8 \mathrm{CB}$ and $8 \mathrm{OCB}$ doped with a small amount of the chiral molecule R 811 . The angular velocity of the droplets strongly depend on their size and on the concentration of chiral molecules. The Lehmann coefficient is estimated by using three different methods. Our results are consistent with a Lehmann coefficient proportional to the concentration of chiral molecules. We additionally show the existence of a critical size of the droplets below which they change texture and stop rotating.
\end{abstract}

PACS. 61.30.-v Liquid crystals - 65.40.De Thermal expansion; thermomechanical effects - 05.70.Ln Nonequilibrium and irreversible thermodynamics

\section{Introduction}

Recently, we reproduced the original Lehmann experiment $[1,2]$ on the continuous rotation of cholesteric droplets subjected to a temperature gradient [3]. We used for that experiment a cholesteric mixture composed of $50 \%$ (by weight) of the liquid crystal 8OCB (4-n-octyloxy-4' cyano biphenyl) and of $50 \%$ of the chiral molecule CC (cholesteryl chloride). In this particular system, the twist vanishes and changes sign with the temperature a few degrees below the clearing temperature (compensated mixture). For this reason, one might expect that the value found for the Lehmann coefficient in this mixture was not characteristic of usual cholesteric liquid crystals. To check this point, we performed new experiments with a common cholesteric

\footnotetext{
a e-mail: patrick.oswald@ens-lyon.fr
} 
mixture and studied the role of the concentration of chiral molecules.

The plan of the article is the following. In Section 2, we present the new cholesteric mixture and we show how to simply estimate at the clearing temperature the helix twist power (HTP) of the chiral molecule chosen. In Section 3 , we report systematic measurements of the period of rotation of the cholesteric droplets as a function of their diameter, the temperature gradient and the concentration of chiral molecules. A first estimate of the Lehmann coefficient is given. In Section 4, we describe the action of an electric field. This yields a second estimate of the Lehmann coefficient. In Section 5, we show the existence of a critical radius below which the droplets change texture and stop rotating. From this observation, we deduce a third estimate of the Lehmann coefficient. Finally, we draw conclusions in Section 6.

\section{The cholesteric mixture: phase diagram and HTP of the chiral molecule at the clearing temperature}

The nematic liquid crystal chosen is an eutectic mixture (EM) of 8CB (4-n-octy-4' cyanobiphenyl from Frinton Laboratories, Inc) and of 8OCB (4-n-octyloxy-4' cyanobiphenyl from Synton Chemicals GmbH \& Co) in the mass proportion of $57: 43$ [4]. This mixture has the advantage of melting at a convenient temperature allowing us to impose a large temperature gradient and of crystallizing at low temperature $\left(5^{\circ} \mathrm{C}\right)$. This liquid crystal is doped with the chiral molecule R811 (from Merck Ltd). The phase diagram of the mixture EM/R811 is shown in Fig. 1. It is worth to mention that the freezing range is close to $0.7^{\circ} \mathrm{C}$ and changes little with the concentration of chiral molecules.

In order to measure the HTP of the chiral molecule R811 at the clearing temperature, we must measure the helical pitch $P$ of the cholesteric phase as a function of the mass concentration $C$ of chiral molecules. We recall that, by definition, $H T P=1 /(C \times P)$. In principle, the HTP is constant at low $C[5]$. The Cano-wedge method is usually used to measure the pitch. It consists of measuring the positions of disclination lines in a wedge sample treated for planar and parallel anchoring. In practice, this method is delicate to carry out, in particular when the pitch is very small, because the sample thickness profile must be known very precisely [6]. For this reason, and because we are mainly interested in a possible variation of the HTP with the concentration of chiral molecules $C$, we used another method easier to carry out. More precisely, we prepared homeotropic samples at concentrations $C=2.11,4.38,5.88$ and 7.9 wt.\% between transparent electrodes (glass plates covered with an ITO layer) and measured for each sample the spinodal voltages (at $10 \mathrm{kHz}) V_{0}$ (resp. $V_{3}$ ) below which (resp. above which) the homeotropic "nematic" phase (resp. the cholesteric phase) become completely unstable $[7,8]$. Our results are reported in Fig. 2 for samples of thickness $d=10 \mu \mathrm{m}$. We observe that $V_{0}$ and $V_{3}$ are proportional to $C$ which indicates that the HTP does not depend on the concentration 
of chiral molecules up to $10 \%$. Indeed, we know from the theory that in the limit $d \gg P[7,8]$

$$
V_{0}=2 \pi \sqrt{\frac{K_{2}}{K_{32} \varepsilon_{0} \varepsilon_{a}}} d \times H T P \times C
$$

and

$$
V_{3}=\pi^{2} \sqrt{\frac{K_{2}}{\varepsilon_{0} \varepsilon_{a}}} d \times H T P \times C
$$

where $\varepsilon_{0}$ is the permittivity of the vacuum, $\varepsilon_{a}$ the dielectric anisotropy, $K_{2}$ and $K_{3}$ the twist and the bend constants, respectively, and $K_{32}=K_{3} / K_{2}$. From the ratio of the slopes of the two lines in Fig. 2 we find that $K_{32} \approx 2.3$. As for the HTP of the chiral material R811 at the clearing temperature, it can be estimated by tak$\operatorname{ing} K_{2}=1.4 \times 10^{-12} \mathrm{~N}$ and $\varepsilon_{a}=4.6$ (knowing that, at the clearing temperature, $K_{2} \approx 1.2 \times 10^{-12} \mathrm{~N}[9]$ and $\varepsilon_{a} \approx 5[10]$ in $8 \mathrm{CB}$ and $K_{2} \approx 1.6 \times 10^{-12} \mathrm{~N}[9]$ and $\varepsilon_{a}=4$ [11]) in $8 \mathrm{OCB}$ ). This yields HTP $\approx 17 \mu \mathrm{m}^{-1}$ (with a plus sign as we know from Merck that R811 is right-handed). This value is close to that found by Baudry by using the Cano-wedge method in $8 \mathrm{CB}$ at the clearing temperature: $\mathrm{HTP} \approx 16 \mu \mathrm{m}^{-1}[12]$. The important result, here, is that the HTP is constant and is independent of the concentration of chiral molecules (up to $9 \%$ by weight).

\section{Period of rotation of the cholesteric}

\section{droplets: experimental data and first}

\section{estimate of the Lehmann coefficient}

In this section, we present our experimental results after a short description of the experiment. The data are then explained within a simple model from which a first estimate of the Lehmann coefficient is deduced.

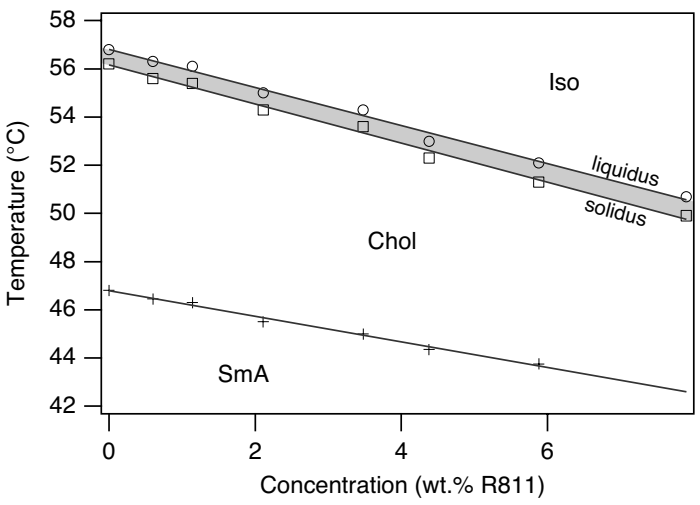

Fig. 1. Phase diagram of the mixture $8 \mathrm{CB} / 8 \mathrm{OCB} / \mathrm{R} 811$. The grey region is a two-phase region.

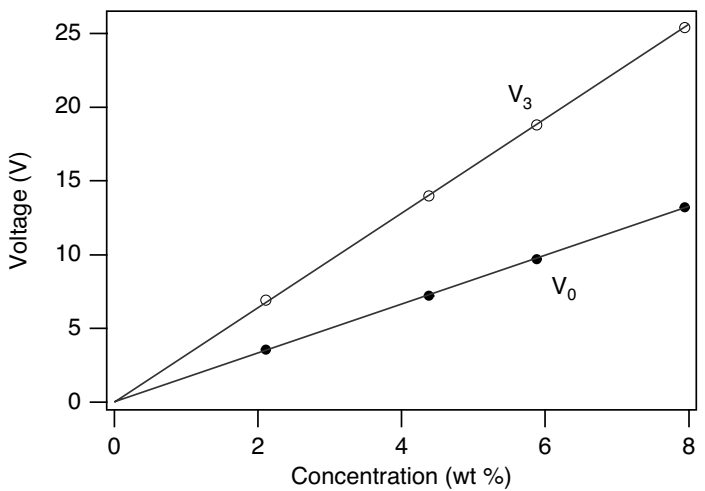

Fig. 2. Spinodal voltages for the homeotropic nematic phase and the cholesteric phase measured at $10 \mathrm{kHz}$ at the solidus temperature as a function of the concentration of R811. $d=$ $10 \mu \mathrm{m}$.

\subsection{Experimental procedure}

All our experiments were performed using $20 \mu \mathrm{m}$-thick samples with the top glass plates treated for planar and sliding anchoring. Such anchoring was obtained by depositing by spin coating a thin layer of the polymercaptan hardener of an epoxy resin (Stucturalit 7) (for more details, see Ref. [13]). The temperature gradient was imposed by sandwiching the sample between two transparent ovens. The setup is described in Ref. [3]. In the following, 

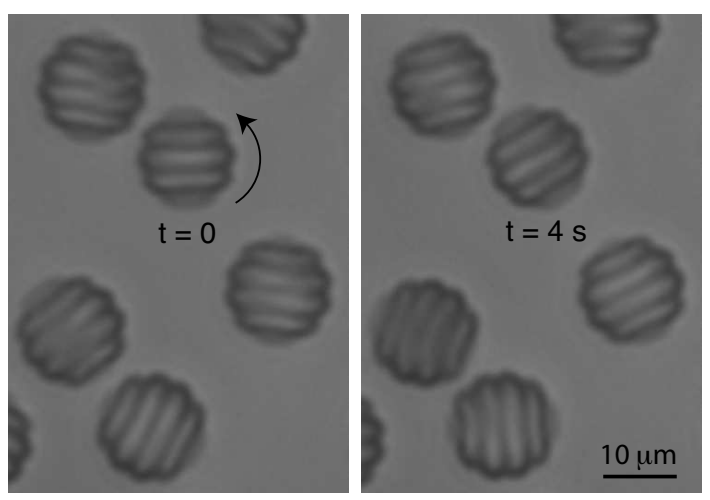

Fig. 3. Droplets observed in natural light rotating anticlockwise under the action of a temperature gradient. The time interval between the two photographs is $4 \mathrm{~s} ; C=1.14 \%$, $\Delta T=10^{\circ} \mathrm{C}$.
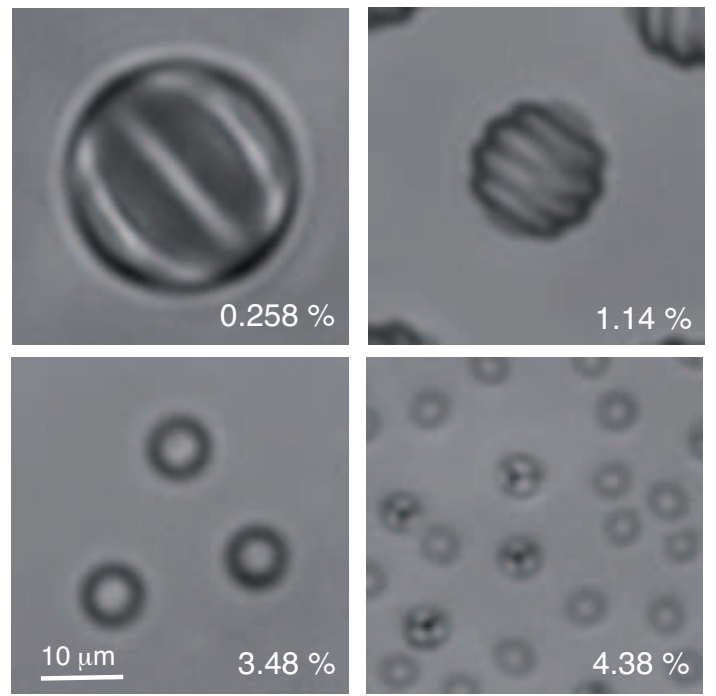

Fig. 4. Droplets observed in natural light for different concentrations. The bands are only visible at small concentration. Note the presence of droplets with three walls starting from the center at large concentration.

we shall denote by $\Delta T$ the difference $T_{b}-T_{t}$ between the temperatures of the bottom and top ovens. Experimentally, the oven temperatures are chosen in order that the sample temperature is close to the liquidus temperature of the cholesteric mixture $\left(\left(T_{b}+T_{t}\right) / 2 \approx T_{l}\right)$. In addition, $\Delta T$ is taken positive in order that the cholesteric droplets form on the top glass plate treated for sliding anchoring. Fig. 3 shows typical droplets observed in natural light in a dilute mixture $(C=0.6 \%)$. As in our previous experiments with the compensated mixture, the droplets are circular and present a banded texture which rotates at constant angular velocity under the action of the temperature gradient. As expected the band wavelength decreases when the concentration of chiral molecules increases and is no longer resolved by the microscope when the concentration is higher than typically $2.5 \%$ (Fig 4). Nevertheless, the droplet rotation can still be detected by adding a polarizer. In such a condition, the droplets appear alternately dark and white in time, which allowed us to measure their period of rotation. We also observed at large concentration droplets with three walls starting from their centers (see the photograph in Fig 4 at $C=4.38 \%$ ). These droplets rotate more slowly than the droplets without defects and rapidly transform into the latter. For this reason, we did not study them systematically.

\subsection{Experimental results}

As in the compensated mixture, the period of rotation $\Theta$ increases when the droplet diameter $D$ increases and decreases when the temperature gradient (or the temperature difference $\Delta T$ ) increases. Fig. 5 shows systematic measurements of the period of rotation measured in a concentrated mixture $(C=7.9 \%)$ as a function of the droplet diameter at four different $\Delta T$. The symbols are the experimental results and the solid lines are polynomial fits to 


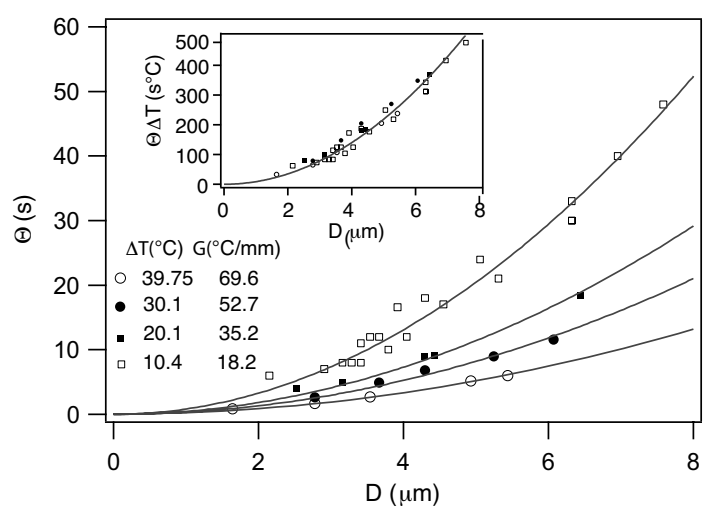

Fig. 5. Period of rotation $\Theta$ as a function of the droplet diameter $D$ measured at four different temperature gradients; $C=7.9 \%$. All points lie on the same curve when we plot $\Theta \Delta T$ as a function of $D$.

the data of the type $a+b D^{2}+c D^{4}$ (this choice will be justified in Section 3.3). We checked that, within experimental error, the period of rotation is inversely proportional to the temperature gradient for each droplet diameter. This can be shown by plotting $\Theta \Delta T$ as a function of $D$ : now all points fall on the same curve as we can see in the inset of Fig. 5 .

We also performed similar measurements at different concentrations of chiral molecules (and different $\Delta T$ ). All our results (normalized to $\Delta T=40{ }^{\circ} \mathrm{C}$ ) are collected in Fig. 6. Again the symbols are the experimental results while the solid lines are polynomial fits to the data. A surprising point is that for a given radius, the period of rotation is a complicated function of the concentration of chiral molecules. This can be seen in Fig.7 where we plotted the period of rotation measured (or extrapolated from the fit at $C=0.258 \%$ ) at diameter $D=5 \mu \mathrm{m}$ as a function of the concentration. We observe that the period of rota-

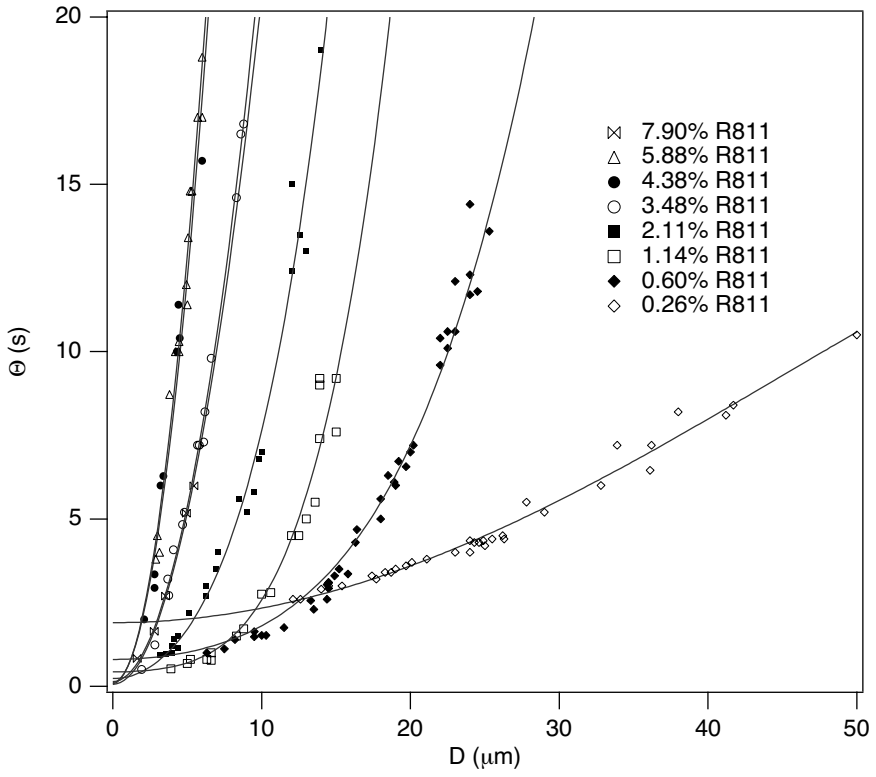

Fig. 6. Period of rotation $\Theta$ as a function of the droplet diameter $D$ measured at $\Delta T=40{ }^{\circ} \mathrm{C}\left(G=70{ }^{\circ} \mathrm{C} / \mathrm{mm}\right)$ for different concentrations of chiral molecules.

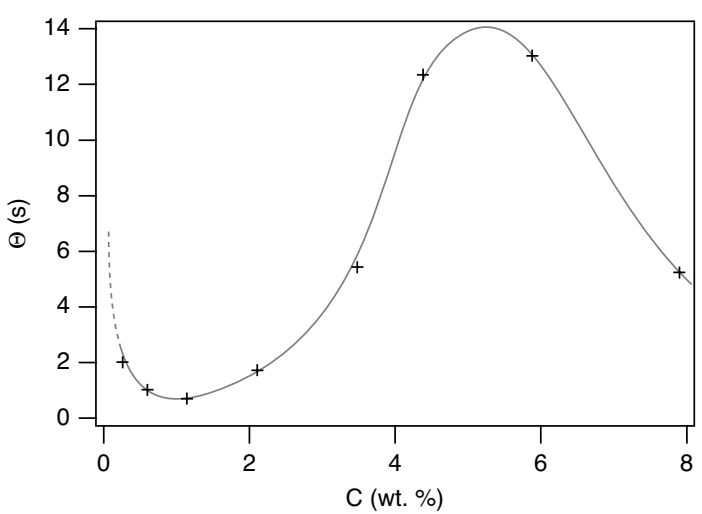

Fig. 7. Period of rotation $\Theta$ as a function of the concentration of chiral molecules $C$ for droplets of diameter $D=5 \mu \mathrm{m}$. The solid line is just a guide for eyes.

tion successively decreases, passes through a minimum, increases, passes through a maximum to finally decrease again when the concentration of chiral molecules increases. This behavior is discussed within a simple model in the next subsection. 


\subsection{Theoretical model and first estimate of the}

\section{Lehmann coefficient}

In Ref. [3] we proposed a simplified model neglecting backflow for explaining the droplet rotation. This model was based on the Leslie theory and consisted of solving the torque equation [14]:

$$
\gamma_{1} \frac{\partial \mathbf{n}}{\partial t}=\mathbf{h}+\nu \mathbf{n} \times \mathbf{G}
$$

where $\mathbf{G}=\nabla T$ is the temperature gradient, $\mathbf{h}=-\frac{\delta f_{e}}{\delta \mathbf{n}}$ is the molecular field (with $f_{e}$ being the usual Frank elastic energy), $\gamma_{1}$ the rotational viscosity and $\nu$ is the Lehmann coefficient. From this equation, we were able to show that the internal texture of the droplets rotate at an angular frequency $\omega=2 \pi / \Theta$ given by

$$
-\frac{\nu G}{\gamma_{1} \omega}=1+\frac{\iiint_{\text {droplet }}\left[\mathbf{e}_{z} \cdot \frac{\partial \mathbf{n}}{\partial \theta} \times \mathbf{n}+\left(\frac{\partial \mathbf{n}}{\partial \theta}\right)^{2}\right] d^{3} r}{\iiint_{\text {droplet }}\left[\mathbf{e}_{z} \cdot \frac{\partial \mathbf{n}}{\partial \theta} \times \mathbf{n}+1-\left(\mathbf{e}_{z} \cdot \mathbf{n}\right)^{2}\right] d^{3} r}
$$

According to this equation, $\omega$ is proportional to $G$, in agreement with experiments, and has the form $\omega=$ $-A \nu G / \gamma_{1}$ where $A$ is a dimensionless (and positive) coefficient which depends on the director field and on the drop geometry. Because the anchoring is planar at the solid surface and rather homeotropic at the cholesteric-isotropic interface, we then proposed that the director field inside the droplets was similar to a modulated TIC. We recall that the TIC is the translationally invariant configuration satisfying the boundary conditions on the two limiting surfaces. Let $k=2 \pi / \lambda$ be the wave vector of the modulation (with $\lambda$ the band wavelength). According to this model, the angular frequency is expressed by a formula of the type

$$
-\frac{\nu G}{\gamma_{1} \omega}=1+\frac{1}{16} k^{2} \varepsilon^{2} D^{2}+O\left(k^{4} \varepsilon^{4} D^{4}\right)
$$

where the angle $\varepsilon$ (supposed to be small) represents the amplitude of the modulation. This formula suggests to fit the measured period of rotation $\Theta$ as a function of the droplet diameter $D$ with a polynomial of the form $a+b D^{2}+c D^{4}$. Note here that in our experiments, $\omega$ and $G$ are of opposite signs (the germs rotate anticlockwise when the temperature gradient points downwards, see Fig. 3), which shows that the Lehmann coefficient is positive. Eq. 5 also shows that the value of $a$ deduced from the fits allows, in principle, a direct measurement of the ratio $\nu / \gamma_{1}$ and, thus, of the Lehmann coefficient $\nu$ providing that $\gamma_{1}$ is known. It turns out that, in practice, $a$ is very small and almost impossible to determine except for the experimental curve obtained at the smallest concentration $C=0.258 \%$. In this case, we find that $a \approx 1.9 \mathrm{~s}$ (see Fig. 6). According to Eq. $5, \frac{\nu}{\gamma_{1}}=\frac{2 \pi}{a G}$ where $G$ is the temperature gradient explicitly given by $G=\frac{\kappa_{g}}{\kappa_{L C}} \frac{\Delta T}{4 e}$, denoting the thickness of the glass plates (one for each oven and two for the sample) by $e$ and the conductivity of the glass (resp. of the liquid crystal) by $\kappa_{g}$ (resp. $\kappa_{L C}$ ). In practice, $e=1 \mathrm{~mm}$ and $\frac{\kappa_{g}}{\kappa_{L C}} \approx 7[3]$. From these values and the value of $a$ given above we can estimate the value of $\nu / \gamma_{1}$ for the more dilute mixture $\frac{\nu}{\gamma_{1}}(C=0.258 \%) \approx 510^{-5} \mathrm{~s}^{-1} \mathrm{~K}^{-1} \mathrm{~m}$. Unfortunately, it was impossible to do the same thing for the larger concentrations because the experimental points are too much dispersed. On the other hand, we can reasonably assume that, at small concentrations, the Lehmann coefficient is proportional to $C$. Doing this, we can fit all 
the other experimental curves of Fig. 6 by fixing $a$, which is calculated by assuming that it is proportional to $C$ and equal to 1.9 s at $C=0.258 \%$. This procedure allows us to fit all the curves quite well, but this just shows that the assumption $\nu \propto C$ is not incompatible with our experimental data.

Another point concerns the behavior of the period of rotation as a function of the concentration at a given radius (see Fig. 7 plotted for $R=5 \mu \mathrm{m}$ ). From Eq. 5 , we can predict that

$$
\Theta \propto \frac{1+\alpha C^{2}+\beta C^{4}}{C}
$$

where $\alpha$ and $\beta$ are two constants by assuming that $\nu \propto C$ and $k \propto q \propto C$ (the HTP is constant). According to this equation, the period of rotation must rapidly decrease from infinity when $C=0$ (the germs stop rotating when there is no chiral molecules), pass through a minimum and then increase again when the concentration increases. This behavior is indeed observed experimentally at low concentrations $(C<4 \%$ typically) and explains easily in terms of a competition between the Lehmann torque $(\propto C)$ and the viscous torque (independent of $C$ when $\lambda \gg D$ and proportional to $C^{2}$ when $\lambda \ll D$, with $\lambda$ the band wavelength). On the other hand, this model fails to explain the saturation and the subsequent decrease of $\Theta$ observed at large $C$. One explanation could be that $q$, and consequently the dissipation, saturate when $C$ increases (above $5 \%$ ) while the Lehmann torque continues to increase, but we have shown in Section 2 that the HTP is constant in the whole range of concentration investigated. So this explanation must be rejected. Another explanation comes from the choice of the director field used in Ref. [3] to calculate the integrals in Eq. 4. Indeed, we explicitly assumed in this model that the TIC was modulated over the whole thickness of the droplet. Such an assumption is true if the cholesteric pitch is comparable to the droplet thickness. By contrast, this assumption is wrong if the pitch is smaller than the drop thickness, which should arise at large concentration. In this limit, the TIC only deforms and modulates close to the surface in contact with the isotropic liquid (this result was already shown in Ref. [15]). As a result the Lehmann torque increases faster than the viscous torque when the concentration increases, which qualitatively explains why the droplets accelerate again (their period of rotation decreases) at large concentrations of chiral molecules.

To conclude this section, let us give a first estimate of the Lehmann coefficient at concentration $C=0.6 \%$ (two other estimates of this coefficient, at this concentration, will be given in the next two sections). According to the literature, $\gamma_{1}$ is of the order of $0.02 \mathrm{~Pa} \mathrm{~s}$ in both $8 \mathrm{CB}$ and 8OCB at the transition temperature [16]. Assuming $\nu \propto C$ and using the value of $\nu / \gamma_{1}$ given above for $C=0.258 \%$, we calculate by taking $\gamma_{1}=0.02 \mathrm{~Pa} \mathrm{~s}$ :

$$
\nu(C=0.6 \%) \sim 2 \times 10^{-6} \mathrm{~kg} \mathrm{~K}^{-1} \mathrm{~s}^{-2}
$$

In the next Section, we show another method to estimate this coefficient. 

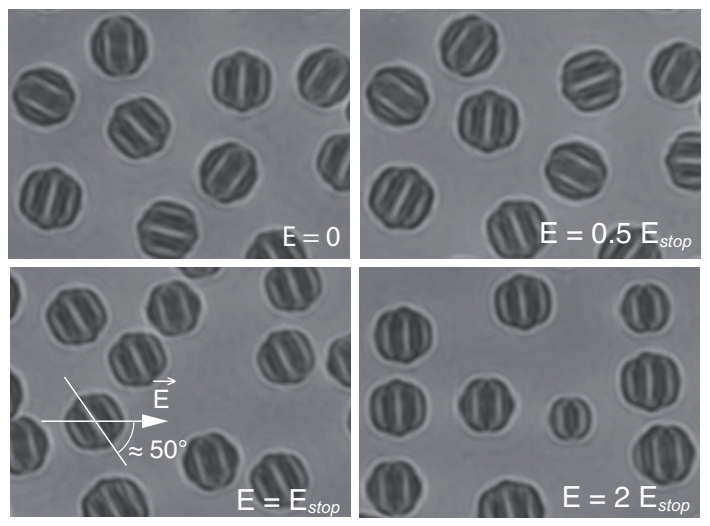

Fig. 8. Droplets subjected to an electric field. At zero or small electric field, they are randomly oriented because they rotate. At $E_{\text {stop }}$ and above, the droplets are immobile and are all oriented in the same way with respect to the electric field (parallel to the long side of the pictures). $C=0.6 \%, \Delta T=10{ }^{\circ} \mathrm{C}$ $\left(G=17.5^{\circ} \mathrm{C} / \mathrm{mm}\right)$.

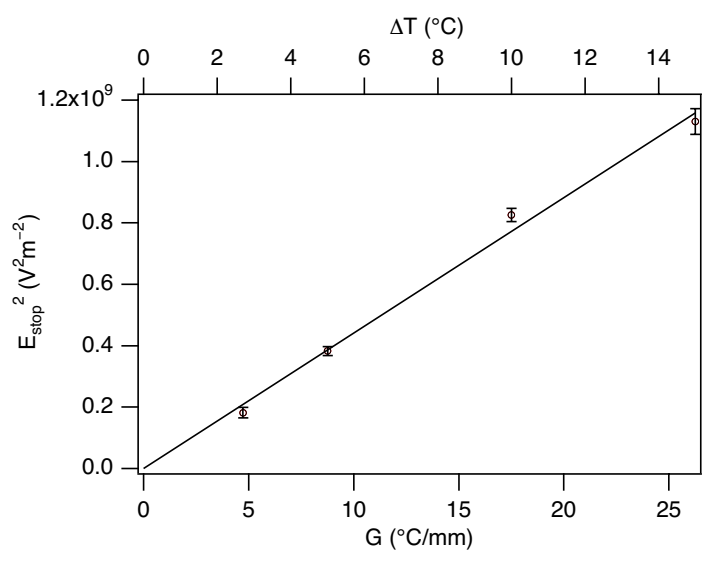

Fig. 9. Square of the critical voltage as a function of the temperature difference (proportional to $G$ ).

\section{Action of an electric field: second estimate of the Lehmann coefficient}

In Ref. [3], we showed that it was possible to stop the droplet rotation by applying a horizontal electric field. We conducted a similar experiment by applying an AC electric field $(10 \mathrm{kHz})$ between two parallel metallic wires stretched across the sample. The wires, also used as spacers between the two glass plates, were $20 \mu \mathrm{m}$ in diameter and separated by a gap of $2.4 \mathrm{~mm}$. Our experiment was performed with the mixture of concentration $C=0.6 \%$. We observed that above some critical electric field $E_{\text {stop }}$ all the droplets stop rotating. At this voltage the bands are oriented at about $45^{\circ}$ with respect to the electric field (Fig. 8). This angle results from a competition between the electric torque which tends to orient the bands perpendicularly to the electric field and the thermomechanical Lehmann torque. In Ref. [3], we gave without demonstration:

$$
E_{\text {stop }}^{2} \approx \frac{2 G \nu}{\varepsilon_{0} \varepsilon_{a}}
$$

This result can be obtained by equilibrating the torques acting on the director. Indeed, let us denote by $\theta$ and $\phi$ the azimuthal and zenithal angles of the director with respect to the $z$ and $x$ axes, respectively (the temperature gradient is supposed to be parallel to $z$ and the electric field parallel to $y$ ). The director experiences three torques: an elastic torque of general expression $\mathbf{n} \times \mathbf{h}$ where $\mathbf{h}$ is the elastic molecular field, the electric torque of expression $\varepsilon_{0} \varepsilon_{a}(\mathbf{n} \times \mathbf{E}) \mathbf{n} \cdot \mathbf{E}$, and the Lehmann torque of expression $\nu \mathbf{n} \times(\mathbf{n} \times \mathbf{G})$. Without temperature gradient and electric field, the director field inside the droplets is such that $\mathbf{n} \times \mathbf{h}=0$. Experimentally, the Lehmann and electric torques are small and do not visibly deform the director field inside the droplets. We can thus assume, as a first approximation, that the director field remains unchanged when the electric field and the temperature gradient are applied. This is justified as the applied voltage is always 
much smaller than that $V_{3}$ required to unwind the helical structure (of the order of $2 \times 10^{5} \mathrm{~V} / \mathrm{m}$ at this concentration). Doing this assumption, the torque equation reduces to equilibrating the Lehmann and electric torques. This yields $\frac{1}{2} E^{2} \varepsilon_{0} \varepsilon_{a} \sin ^{2} \theta \sin 2 \phi=\nu G \sin ^{2} \theta$. This equation immediately shows that the smallest value of the electric field $E_{\text {stop }}$ for which equilibrium is possible, is well given by Eq. 7, irrespective of the value of angle $\theta$, when $\phi=45^{\circ}$ in average and in agreement with experiment (see Fig. 8).

According to Eq. 7, $E_{\text {stop }}^{2}$ must be proportional to $G$. We checked this point experimentally as we can see in Fig. 9. From the slope of this curve and the previous equation, we calculate

$$
\nu(C=0.6 \%) \sim 1 \times 10^{-6} \mathrm{kgK}^{-1} \mathrm{~s}^{-2}
$$

by taking $\varepsilon=4.6$.

This value is twice as small as that given in the previous section, but it is of the same order of magnitude.

\section{Textural change of cholesteric droplets:}

\section{third estimate of the Lehmann coefficient}

We observed that, in dilute samples $(C=0.258 \%$ and $C=0.6 \%$, the droplets change texture below some critical diameter and do not rotate anymore. This is visible in Fig. 10 where we can see the two types of droplets. While the banded one rotates, any of the others show visible rotation, even when one adds a polarizer. Observations between crossed polarizers of these new droplets show Maltese crosses which rotate in the same direction as the polarizers when the latter are rotated (Fig. 11).

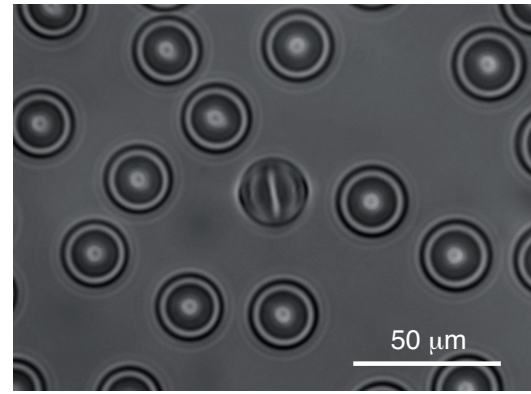

Fig. 10. Two types of droplets coexisting in the same sample (observation in natural light). All the droplets, the radii of which are very close to $R_{\max }$, are immobile except the central banded one which is rotating; $C=0.258 \%, \Delta T=5{ }^{\circ} \mathrm{C}(G=$ $\left.8.7^{\circ} \mathrm{C} / \mathrm{mm}\right)$.

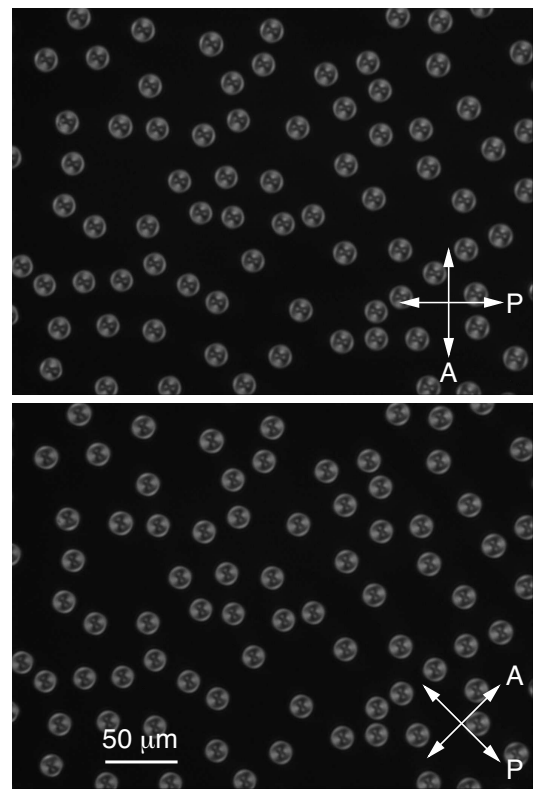

Fig. 11. Static droplets observed between crossed polarizers; $C=0.258 \%, \Delta T=5{ }^{\circ} \mathrm{C}\left(G=8.7{ }^{\circ} \mathrm{C} / \mathrm{mm}\right)$.

This indicates that their director field is invariant by rotation about an axis perpendicular to the glass plates and has the topology of a $S=+1$ disclination line. Another important observation is that the Maltese crosses do not rotate inside the droplets when the sample is subjected to a temperature gradient. This means that the director field 
is frozen inside the droplets in spite of the presence of a Lehmann torque. This observation is easily understandable. Indeed, let us assume that the director rotates. In this case, the disclination would successively pass from a circular configuration to a radial one. In practice, these two configurations are not energetically equivalent, the radial configuration being less energetic than the circular one because of the elastic anisotropy $\left(K_{3}\right.$ is usually larger than $\left.K_{1}[9]\right)$. As a consequence, the rotation is only possible if the Lehmann torque can perform a work equal to the energy difference between the two configurations when the director rotates by $\pi / 2$. Let $R$ be the radius of the droplet and $h$ its thickness. According to this criterion, the texture can rotate only if the following inequality is satisfied:

$$
\nu G \frac{\pi}{2} \pi R^{2} h \geq \pi\left(K_{3}-K_{1}\right) \ln \frac{R}{r_{c}} h
$$

This defines a maximum radius $R_{\max }$ below which the droplet texture can no longer rotate (the texture is pinned in its radial and less energetic configuration):

$$
R_{\max }=\sqrt{\frac{2}{\pi} \frac{K_{3}-K_{1}}{\nu G} \ln \left(\frac{R}{r_{c}}\right)}
$$

where $r_{c}$ is a core radius of molecular size (typically $100 \stackrel{\circ}{A}$ ).

We measured $R_{\max }$ for different temperature gradients (or $\Delta T)$ and concentrations $(C=0.258 \%$ and $C=0.6 \%)$. Our results are given in the table below.

\begin{tabular}{lll}
\hline$R_{\max }$ & $G$ & $C$ \\
\hline 11.4 & 5 & 0.258 \\
6.3 & 15 & 0.258 \\
5.2 & 10 & 0.6 \\
3.8 & 15 & 0.6 \\
2.8 & 30 & 0.6 \\
\hline
\end{tabular}

From these measurements, we can check that the product $R_{\max }^{2} \Delta T C$ is approximately constant as stated by Eq. 8 under the assumption that $\nu$ is proportional to $C$ : $R_{\max }^{2} \Delta T C=150 \pm 20 \mu \mathrm{m}^{2} \mathrm{~K}$ wt.\%. This average value and Eq. 8 allow us to calculate a new estimate of the Lehmann coefficient knowing that in both $8 \mathrm{CB}$ and $8 \mathrm{OCB}, K_{3}-K_{1}$ is of the order of $2 \times 10^{-13} \mathrm{~N}$ at the transition temperature $[9]:$

$$
\nu(C=0.6 \%) \sim 2 \times 10^{-6} \mathrm{kgK}^{-1} \mathrm{~s}^{-2}
$$

This value is in agreement with the two previous estimates.

\section{Conclusion}

In conclusion, we evidenced experimentally the Lehmann rotation of cholesteric droplets in a dilute cholesteric solution of the chiral molecule R811 dissolved in a nematic eutectic mixture of $8 \mathrm{CB}$ and $8 \mathrm{OCB}$. Droplets are observed at the coexistence temperature between the cholesteric phase and the isotropic liquid. As in previous experiments [3], we observed that the angular velocity was strongly dependent on the director field configuration inside the droplets and showed that the droplet rotation can be stopped by apply- 
ing an AC electric field. We additionally evidenced a critical radius below which the droplets change their configuration (from a banded texture to a rotationally invariant one) and stop rotating. We estimated the Lehmann coefficient in three different ways and obtained the values of the order of $10^{-6} \mathrm{kgK}^{-1} \mathrm{~s}^{-2}$ for a concentration of $0.6 \%$. We emphasize that this value is comparable with that found previously at the clearing temperature in the compensated mixture (8OCB doped with $50 \%$ by weight of CC). This result could seem surprising as the concentration of chiral molecules is almost 100 times larger in the compensated mixture than in the present mixture (50\% against $0.6 \%)$. On the other hand, the pitches of the two mixtures are similar as $P \approx 10 \mu \mathrm{m}$ in the $8 \mathrm{CB} / 80 \mathrm{CB} / \mathrm{R} 811$ mixture at $C=0.6 \%$ while $P \approx 6 \mu \mathrm{m}$ in the compensated mixture at the clearing temperature (value obtained by extrapolating the curve $q(T)$ given in Ref. [17] to this temperature). This result shows that the equilibrium twist $q$ and the Lehmann coefficient $\nu$ are strongly correlated even if they are not strictly proportional to each other at large concentrations of chiral molecules (indeed, we have shown previously that in the $8 \mathrm{OCB} / \mathrm{CC}$ mixture $\nu \neq 0$ at the compensation temperature at which $q=0$ ). On the other hand, our present data suggest that, as expected, $\nu$ is proportional to $q$ in the limit of dilute mixtures.

The next step is now to measure the Lehmann coefficient below the clearing temperature. This can be done as in Ref. [18] by observing directly the rotation of the director in samples treated on both glass plates for planar and sliding anchoring. We already performed this experi- ment just below the solidus temperature with the mixture at the concentration of $0.6 \%$. As expected, we observed the Lehmann rotation and found that, as in the compensated mixture, the Lehmann coefficient seems to rapidly decrease in the close vicinity of the phase transition when the temperature decreases, contrary to the equilibrium twist. On the other hand, our measurements are still too much dispersed to be published. We are currently working on this problem.

\section{Acknowledgments}

We thank A. Zywocinski for the purification of the $80 \mathrm{CB}$ and careful rereading of the manuscript and A. Dequidt for discussions.

\section{References}

1. O. Lehmann, Ann. Physik 2, (1900) 649.

2. O. Lehmann, Flüssige Kristalle und ihr Scheinbares Leben (Verlag von Leopold Voss, Leipzig 1921).

3. P. Oswald, A. Dequidt, Phys. Rev. Lett. 100, (2008) 217802.

4. S. Delenclos, C. Kolinsky, S. Longuemart, A. Hadj Sahraoui, J. M. Buisine, J. Therm. Anal. Cal. 70, (2002) 549.

5. P.-G. de Gennes, The Physics of Liquid Crystals (Clarendon Press, Oxford 1974).

6. T. Kosa, V. H. Bodnar, B. Taheri, P. Palffy-Muhoray, Mol. Cryst. Liq. Cryst. 369, (2001) 129.

7. P. Oswald, P. Pieranski, Nematic and Cholesteric Liquid Crystals: Concepts and Physical Properties Illustrated by Experiments (Taylor \& Francis, CRC press, Boca Raton 2005). 8. P. Oswald, J. Baudry, S. Pirkl, Phys. Rep. 60, (2000) 67. 
9. N. V. Madhusudana, R. Pratibha, Mol. Cryst. Liq. Cryst.

89, (1982) 249 .

10. B. R. Ratna, S. Shashidhar, Pramana 6, (1976) 278.

11. J. Jadżyn, G. Czechowski, Liq. Cryst. 4, (1989) 157.

12. J. Baudry, PhD thesis, Statique et dynamique des doigts cholestériques sous champ électrique, École Normale Supérieure de Lyon, 1999.

13. P. Oswald, A. Dequidt, A. Żywociński, Phys. Rev. E 77, (2008) 061703.

14. F.M. Leslie, Proc. Roy. Soc. London A 307, (1968) 359.

15. J. Baudry, M. Brazovskaia, L. Lejcek, P. Oswald, S. Pirkl, Liq. Cryst. 21, (1996) 893.

16. H. Kneppe, F. Schneider, N. K. Sharma, J. Chem. Phys. 77, (1982) 3203 .

17. A. Dequidt, P. Oswald, Europhys. Lett. 80, (2007) 26001.

18. P. Oswald, A. Dequidt, Europhys. Lett. 83, (2008) 16005. 